\title{
Analytic Structure of Three-Mass Triangle Coefficients
}

\author{
N. E. J. Bjerrum-Bohr ${ }^{1}$, David C. Dunbar ${ }^{2}$ and Warren B. Perkins ${ }^{2}$ \\ ${ }^{1}$ Institute for Advanced Study, \\ Princeton, NJ 08540, USA \\ ${ }^{2}$ Department of Physics, \\ Swansea University, \\ Swansea, SA2 8PP, UK
}

\begin{abstract}
Three-mass triangles" are a class of integral functions appearing in oneloop gauge theory amplitudes. We discuss how the complex analytic properties and singularity structures of these amplitudes can be combined with generalised unitarity techniques to produce compact expressions for three-mass triangle coefficients. We present formulae for the $\mathcal{N}=1$ contributions to six and seven gluon scattering and describe how these can be used as the starting point to recursively obtain these coefficients in the $n$-point NMHV case.
\end{abstract}

KEYWORDs: NLO computations, Supersymmetric gauge theory. 


\section{Contents}

1. Introduction 1

2. Organisation of Amplitudes 3

3. Singularity Structure of Six-Point Three-Mass Triangles 4

3.1 Infra-Red Singularities 5

3.2 Spurious Singularities 6

4. Analytic evaluation of the Three-Mass Triangle Coefficients 10

5. Seven-Point Results 14

6. NMHV Three-Mass Triangles From Recursion 17

7. Conclusions 19

8. Acknowledgments 20

\section{Introduction}

A general $n$-point one-loop amplitude in a massless theory such as QCD can be expanded in terms of integral functions,

$$
A_{n}^{1-\text { loop }}=\sum_{i \in \mathcal{C}} c_{i} I_{4}^{i}+\sum_{j \in \mathcal{D}} d_{j} I_{3}^{j}+\sum_{k \in \mathcal{E}} e_{k} I_{2}^{k}+R
$$

where $c_{i}, d_{i}, e_{i}$ and $R$ are rational functions and the $I_{4}, I_{3}$, and $I_{2}$ are scalar box, triangle and bubble functions respectively. The mathematical form of these integral functions depends on whether the momenta flowing into a vertex are null (massless) or not (massive). This decomposition suggests a "divide and conquer" approach to evaluating one-loop amplitudes where different techniques are used to evaluate the different types of coefficient.

In principle, traditional Feynman diagram techniques, combined with reduction strategies can be used to compute the integral coefficients $[1,2]$. Considerable progress 
has been made in implementing this strategy, however the degree of complexity rises rapidly with the number of external legs and the current state of the art is in the computation of five and six-point amplitudes (see for example [3,4]).

Alternate approaches based on the physical properties of amplitudes have proved competitive or superior, particularly in computing amplitudes with enhanced symmetry, such as those appearing in supersymmetric theories, or for amplitudes with particular helicity configurations. Two particularly powerful methods have been those based on unitarity and factorisation. The conjectured duality between the perturbation theory of gauge theories and string theory [5] has provided added insight to these approaches, particularly with respect to complex factorisation.

The unitarity method [6,7], combined with a knowledge of a basis set of integral functions for an amplitude, provides a systematic way of calculating loop amplitudes. Two-particle cuts provide sufficient information to identify many of the coefficients in eqn. (1.1) particularly in cases where the amplitude is "cut-constructible" [6-11]. In addition, extending to $D$-dimensional unitarity, in principle, provides the information to calculate the rational parts [12]. Three and four particle cuts may also be used to identify coefficients of triangle and box functions $[8,13,14]$. For the box coefficients, $c_{i}$, quadruple cuts [13] are particularly simple since the loop momentum integration is frozen by the insertion of four $\delta$-functions.

The analytic structure of the cut integrals appearing in the unitarity method can also be exploited to obtain coefficients. For example, the "holomorphic anomaly" provided an insight into the differing analytic properties of various integral functions in the two-particle cuts [15-17]. Various techniques have been developed to identify the integral coefficients based on the analytic structure of the integrand of the cut $[9$, $10,18]$.

In this paper we explore some recent suggestions for evaluating the coefficients of the "three-mass triangle" integral functions $I_{3}^{3 m}\left(K_{1}, K_{2}, K_{3}\right)\left(K_{i}^{2} \neq 0\right)$ by using the analytic structure of the triple cut. In ref. [19] an algebraic technique was presented for obtaining these coefficients. In this paper we review and refine this technique and present a version that uses a single contour integration.

Although we can divide the amplitude into separate coefficients, in general, different integral coefficients are related by a rich web of "spurious singularities". These are singularities that are not present in the full amplitude but which appear in individual coefficients. This structure is particularly rich for the three-mass triangles. We explore and use this to obtain compact expressions for these coefficients in the $\mathcal{N}=1$ contributions to six gluon scattering. These provide alternate forms to those originally calculated in [9].

The expressions for the three-mass triangle coefficients we obtain are in a suitable form to be used as starting points for recursively evaluating the coefficients for higher 
point functions [20]. We demonstrate this technique by evaluating the coefficients of the three-mass triangles appearing in the $\mathcal{N}=1$ contributions to seven gluon scattering and describe how the coefficients may be obtained for the $n$-point "nextto-MHV" (NMHV) contributions.

\section{Organisation of Amplitudes}

For one-loop amplitudes with external gluons in QCD it is convenient to decompose the contribution from gluons circulating in the loop into pieces corresponding to complex scalars or supersymmetric multiplets circulating in the loop,

$$
A_{n}^{1-\text { loop }}=A_{n}^{\mathcal{N}=4}-4 A_{n}^{\mathcal{N}=1 \text { chiral }}+A_{n}^{\text {scalar }} .
$$

The $A_{n}^{\mathcal{N}=4}$ component consists entirely of box integrals. The terms we look at in this

paper are the $A_{n}^{\mathcal{N}=1 \text { chiral }}$ contributions. These amplitudes may contain any of the integral functions (but not rational terms). Furthermore we consider the amplitude to be colour ordered and focus our attention on the leading in colour component from which the full amplitude can be obtained [6,21].

The integrals appearing in the amplitude may be box, triangle or bubble functions. We are interested in the contributions of three-mass triangles. The relevant integral function is defined by,

$$
I_{3}^{3 m}=i(4 \pi)^{2-\epsilon} \int \frac{d^{4-2 \epsilon} p}{(2 \pi)^{4-2 \epsilon}} \frac{1}{p^{2}\left(p-K_{1}\right)^{2}\left(p+K_{2}\right)^{2}},
$$

and can be written as $[22,23]$,

$$
I_{3}^{3 m}=\frac{i}{\sqrt{\Delta_{3}}} \sum_{j=1}^{3}\left[\operatorname{Li}_{2}\left(-\left(\frac{1+i \delta_{j}}{1-i \delta_{j}}\right)\right)-\operatorname{Li}_{2}\left(-\left(\frac{1-i \delta_{j}}{1+i \delta_{j}}\right)\right)\right]+\mathcal{O}(\epsilon),
$$

where,

$$
\begin{aligned}
& \delta_{1}=\frac{K_{1}^{2}-K_{2}^{2}-K_{3}^{2}}{\sqrt{\Delta_{3}}}, \\
& \delta_{2}=\frac{K_{2}^{2}-K_{1}^{2}-K_{3}^{2}}{\sqrt{\Delta_{3}}}, \\
& \delta_{3}=\frac{K_{3}^{2}-K_{1}^{2}-K_{2}^{2}}{\sqrt{\Delta_{3}}},
\end{aligned}
$$

and

$$
\Delta_{3} \equiv-\left(K_{1}^{2}\right)^{2}-\left(K_{2}^{2}\right)^{2}-\left(K_{3}^{2}\right)^{2}+2 K_{1}^{2} K_{2}^{2}+2 K_{1}^{2} K_{3}^{2}+2 K_{2}^{2} K_{3}^{2} .
$$

The other integral functions we will encounter can be obtained in many places e.g. [7]. The one-mass triangles depend only on the momentum invariant of the massive leg, $K^{2}$,

$$
I_{3}^{1 m}\left(K^{2}\right)=\frac{r_{\Gamma}}{\epsilon^{2}}\left(-K^{2}\right)^{-1-\epsilon} \equiv G\left(K^{2}\right)
$$


where $r_{\Gamma} \equiv \Gamma(1+\epsilon) \Gamma^{2}(1-\epsilon) / \Gamma(1-2 \epsilon)$. The two-mass triangle integral,

$$
I_{3}^{2 m}\left(K_{1}^{2}, K_{2}^{2}\right)=\frac{r_{\Gamma}}{\epsilon^{2}} \frac{\left(-K_{1}^{2}\right)^{-\epsilon}-\left(-K_{2}^{2}\right)^{-\epsilon}}{\left(-K_{1}^{2}\right)-\left(-K_{2}^{2}\right)},
$$

can be expressed as one-mass triangle functions,

$$
I_{3}^{2 m}\left(K_{1}^{2}, K_{2}^{2}\right)=\frac{1}{\left(-K_{1}^{2}\right)-\left(-K_{2}^{2}\right)}\left(G\left(K_{1}^{2}\right)-G\left(K_{2}^{2}\right)\right)
$$

and we can drop these functions from our basis of integral functions in favour of $G\left(K^{2}\right)$ functions. The box functions may be found in many places, for example ref. $[23,6]$. We need the form of one of these, namely the integral function where two adjacent legs are massless; the so-called "two-mass hard" function. If $k_{1}$ and $k_{2}$ are the null legs, defining $S \equiv\left(k_{1}+k_{2}\right)^{2}$ and $T=\left(k_{2}+K_{3}\right)^{2}$ we have,

$$
\begin{aligned}
& I_{4}^{2 m h}=\frac{-2 r_{\Gamma}}{S T}\left\{-\frac{1}{\epsilon^{2}}\left[(-S)^{-\epsilon}+(-T)^{-\epsilon}-\left(-K_{3}^{2}\right)^{-\epsilon}-\left(-K_{4}^{2}\right)^{-\epsilon}\right]\right. \\
& \left.-\frac{1}{2 \epsilon^{2}} \frac{\left.\left(-K_{3}^{2}\right)^{-\epsilon}\left(-K_{4}^{2}\right)\right)^{-\epsilon}}{(-S)^{-\epsilon}}+\frac{1}{2} \ln ^{2}\left(\frac{S}{T}\right)+\operatorname{Li}_{2}\left(1-\frac{K_{3}^{2}}{T}\right)+\operatorname{Li}_{2}\left(1-\frac{K_{4}^{2}}{T}\right)\right\} .
\end{aligned}
$$

The coefficients of the integral functions will be expressed as rational functions of spinor inner-products [24], $\langle j l\rangle \equiv\left\langle k_{j}^{-} \mid k_{l}^{+}\right\rangle,[j l] \equiv\left\langle k_{j}^{+} \mid k_{l}^{-}\right\rangle$, where $\left|k_{i}^{ \pm}\right\rangle$is a massless Weyl spinor with momentum $k_{i}$ and chirality \pm . We use notation where,

$$
\left\langle k_{a}^{+}\left|K_{b c d}\right| k_{e}^{+}\right\rangle \equiv\left[a\left|K_{b c d}\right| e\right\rangle=[a b]\langle b e\rangle+[a c]\langle c e\rangle+[a d]\langle d e\rangle
$$

As in twistor-space studies we define,

$$
\lambda_{i}=\left|k_{i}^{+}\right\rangle, \quad \tilde{\lambda}_{i}=\left|k_{i}^{-}\right\rangle .
$$

\section{Singularity Structure of Six-Point Three-Mass Triangles}

In this section we look at the three-mass triangle integrals found in six-point one-loop gluon scattering amplitudes. The only non-vanishing three-mass triangle coefficients appear in the NMHV amplitudes, of which there are three inequivalent forms:

$$
A\left(1^{-}, 2^{-}, 3^{-}, 4^{+}, 5^{+}, 6^{+}\right), \quad A\left(1^{-}, 2^{-}, 3^{+}, 4^{-}, 5^{+}, 6^{+}\right), \quad A\left(1^{-}, 2^{+}, 3^{-}, 4^{+}, 5^{-}, 6^{+}\right) .
$$

The first of these was calculated in ref. [17] and contains no three-mass triangles, as can be seen from the triple-cuts. The remaining two were computed in ref. [9] using the analytic structure of the two-particle cuts. Although correct (as verified by numerical comparison to a Feynman diagram calculation [3]), these expressions

contain irrational expressions involving the square root of the Gram determinant of 
the three-mass triangle, $\sqrt{\Delta_{3}}$. We produce expressions with the correct singularity structure which explicitly do not contain these irrational terms.

We start by considering the amplitude $A\left(1^{-}, 2^{+}, 3^{-}, 4^{+}, 5^{-}, 6^{+}\right)$. As for any supersymmetric amplitude, the cancellations occurring at one-loop imply that no rational terms appear [7]. Further, by examining the unitarity cuts, we see that only one-mass and two-mass hard boxes appear and, as discussed above, we choose a basis where the two-mass triangles are replaced by one-mass triangle functions, $G\left(K^{2}\right)$. We thus have,

$$
\begin{aligned}
A^{\mathcal{N}=1}\left(1^{-}, 2^{+}\right. & \left., 3^{-}, 4^{+}, 5^{-}, 6^{+}\right)=\sum_{i=1}^{6} c_{i}^{1 m} I_{4}^{1 m i}+\sum_{i=1}^{6} c_{i}^{2 m h} I_{4}^{2 m h i}+\sum_{i=1}^{6} d_{i} G\left(s_{i i+1}\right) \\
& +\sum_{i=1}^{3} d_{i}^{\prime} G\left(t_{i i+1 i+2}\right)+d_{1}^{3 m} I_{3}^{3 m} 1+d_{2}^{3 m} I_{3}^{3 m} 2+\sum_{i=1}^{6} e_{i} I_{2}^{2, i}+\sum_{i=1}^{3} e_{i}^{\prime} I_{2}^{3, i},
\end{aligned}
$$

where both of the three-mass triangles, $I_{3}^{3 m} 1\left(K_{12}, K_{34}, K_{56}\right)$ and $I_{3}^{3 m 2}\left(K_{61}, K_{23}, K_{45}\right)$, appear. The labelling of functions is specified below,

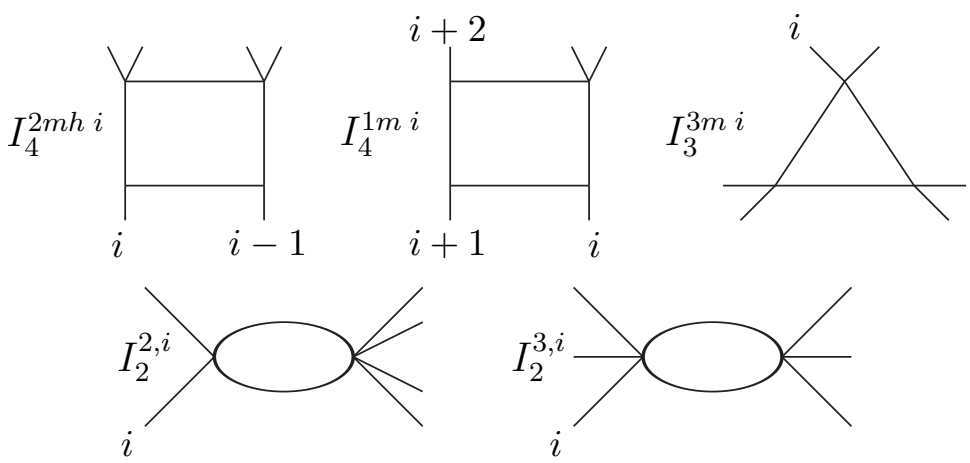

We shall see how much of the amplitude can be reconstructed from the singularity structure: both real and spurious. Our starting point is the box coefficients [25],

$$
\begin{aligned}
c_{1}^{1 m} & =i \frac{[2|K| 5\rangle^{2}[1|K| 5\rangle[3|K| 5\rangle}{[13]^{2}\langle 45\rangle\langle 56\rangle[1|K| 4\rangle[3|K| 6\rangle K^{2}} \times \frac{-s_{12} s_{23}}{2}, \quad K=K_{123}, \\
c_{4}^{2 m h} & =i \frac{[2|K| 5\rangle^{2}[3|K| 5\rangle[2|K| 4\rangle}{[12]\langle 56\rangle[3|K| 6\rangle[1|K| 4\rangle[3|K| 4\rangle^{2}} \times \frac{-s_{34} K^{2}}{2}, \quad K=K_{123} .
\end{aligned}
$$

We will see that these completely determine the coefficients of the one-mass triangles and determine much of the three-mass triangle coefficients.

\subsection{Infra-Red Singularities}

One of the major constraints on the triangle coefficients comes from requiring that the amplitude has the correct infra-red singularities. The box integral functions with 
massless legs and the one-mass triangle functions both contain $\ln \left(K^{2}\right) / \epsilon$ singularities. We then have,

$$
\left[d_{i} G\left(K^{2}\right)+\sum c_{i} I_{4}^{i}\right]_{\ln \left(K^{2}\right) / \epsilon}=0
$$

for the $\mathcal{N}=1$ chiral multiple where such singularities do not appear in the amplitude. These constraints fix the coefficients of the $G\left(K^{2}\right)$ in terms of the box coefficients.

For the $\mathcal{N}=1$ multiplet, one could choose a basis in which the coefficients of the one-mass triangles are zero. There are several options for doing so: firstly one can choose the basis of "six dimensional scalar box functions" as in [17] or one can choose a basis of functions where the IR singularities have been subtracted as in [9]. In the first case the $D=4$ boxes and $D=6$ boxes are related, using the notation of ref. [23],

$$
I_{4}^{D=4}=\frac{1}{2 N_{4}}\left[\sum_{i} \alpha_{i} \gamma_{i} I_{3}^{(i)}+(-1+2 \epsilon) \hat{\Delta}_{4} I_{4}^{D=6}\right],
$$

where $I_{3}^{(i)}$ is the descendant triangle in which the $i$-th propagator is deleted. If we change the basis of box integral functions, the coefficients of the triangles, including the three-mass, are shifted,

$$
d_{i} \longrightarrow d_{i}+\frac{\alpha_{i} \gamma_{i}}{2 N_{4}} c_{b o x}
$$

Although transforming to this basis is instructive, we will continue to use the basis of $D=4$ integral functions.

\subsection{Spurious Singularities}

Looking at the box coefficients we see that factors such as,

$$
\frac{1}{\left[3\left|K_{123}\right| 4\right\rangle^{2}} \text { and } \frac{1}{[13]^{2}}
$$

appear. The first of these is singular when the momenta are arranged such that,

$$
k_{1}^{\mu}+k_{2}^{\mu}=\alpha k_{3}^{\mu}+\beta k_{4}^{\mu},
$$

and such singularities are termed co-planar ${ }^{1}$. These singularities are spurious, meaning they may appear in individual terms within an amplitude but disappear when the entire amplitude is constructed. These coplanar singularities do not cancel amongst the boxes, but cancel between the boxes and the other integral functions. For real momenta these singularities occur when,

$$
t_{123}^{2}+\left(s_{34}-s_{56}-s_{12}\right) t_{123}+s_{56} s_{12}=0 .
$$

\footnotetext{
${ }^{1}$ For real momenta $[4|K| 3\rangle$ and $[3|K| 4\rangle$ vanish simultaneously. However, by continuing to complex momenta we can find a point where only one of them vanishes, e.g. if $k_{1}+k_{2} \sim \lambda_{x} \bar{\lambda}_{3}+\lambda_{4} \bar{\lambda}_{y}$, $[3|K| 4\rangle=0$ but $[4|K| 3\rangle \neq 0$.
} 
At the coplanar singularity the link between the box coefficients and the one-mass triangles implies that the latter also have coefficients with quadratic singularities. However the cancellation of the spurious singularities extends beyond these functions. At this singularity the dilogarithms within the two mass hard function simplify since $s_{12}=\alpha \beta s_{34}, s_{56}=(1+\alpha)(1+\beta) s_{34}$ and $t_{123}=\alpha(1+\beta) s_{34}$, leading to,

$$
I_{4}^{2 m h} \sim\left(\operatorname{Li}_{2}\left(1-\frac{\alpha}{(1+\alpha)}\right)+\operatorname{Li}_{2}\left(1-\frac{1+\beta}{\beta}\right)\right) .
$$

These must cancel against another integral function containing dilogarithms: the three-mass triangle being the only possibility. The cancellation of spurious singularities can thus be expressed as,

$$
\left[c_{i} I_{4}^{i}+d_{i} I_{3}^{1 m i}+d^{3 m} I_{3}^{3 m}\right]_{[a|K| b\rangle=0}=\text { finite } .
$$

This imposes a significant constraint on the three-mass triangle coefficient. One approach is to change basis to one where this cancellation is automatic. This process is essentially the same as that of ref. [8] where the three-mass triangle functions arise in $e^{+}+e^{-} \longrightarrow$ four parton scattering. We can generate this combination using the identity (3.5),

$$
I_{4}^{D=4}-\frac{1}{2 N_{4}}\left[\sum_{i} \alpha_{i} \gamma_{i} I_{3}^{(i)}\right]=(-1+2 \epsilon) \frac{\hat{\Delta}_{4}}{2 N_{4}} I_{4}^{D=6}
$$

For the two-mass hard box we have,

$$
\frac{\hat{\Delta}_{4}}{2 N_{4}}=-2\left(\frac{\operatorname{tr}\left(\not k_{i-1} \not P \not \not_{i} P\right)}{S T^{2}}\right)=-2 \frac{[i|P| i-1\rangle[i-1|P| i\rangle}{s_{i-1 i}\left(P^{2}\right)^{2}} .
$$

Thus $\hat{\Delta}_{4} / 2 N_{4} \longrightarrow 0$ at the coplanar singularity and since $I_{4}^{D=6}$ is finite at this (unphysical) singularity we must have,

$$
I_{4}^{D=4}-\frac{1}{2 N_{4}}\left[\sum_{i} \alpha_{i} \gamma_{i} I_{3}^{(i)}\right] \longrightarrow 0
$$

at the coplanar singularity. Up to a scaling factor this is precisely the $L s_{1}$ function of ref. [8]. As this combination includes a three-mass triangle, the coefficient of this three-mass triangle in the $D=4$ basis must contain a term,

$$
-\frac{\alpha_{i} \gamma_{i}}{2 N_{4}} c_{4}^{2 m h}
$$

which suggests the term,

$$
\frac{-\left[2\left|K_{123}\right| 4\right\rangle\left[2\left|K_{123}\right| 5\right\rangle\left[3\left|K_{123}\right| 5\right\rangle}{\left[1\left|K_{123}\right| 4\right\rangle\left[3\left|K_{123}\right| 4\right\rangle^{2}\left[3\left|K_{123}\right| 6\right\rangle t_{123}} \frac{\left[2\left|K_{123}\right| 5\right\rangle\left(2 s_{12} s_{56}-\left(s_{12}+s_{56}-s_{34}\right) t_{123}\right)}{2\langle 56\rangle[12]}
$$


within $d^{3 m}$. Since the three-mass triangle is the "daughter" of three different twomass-hard boxes, each with a different quadratic singularity, we have three such terms in the coefficient.

This expression gives an amplitude from which the quadratic spurious singularity is absent. However we have introduced a further fictitious singularity : a $t_{123}$ pole. The three-mass triangle should not contain such a pole. We can "fix" this by adding an extra term, giving,

$\frac{-\left[2\left|K_{123}\right| 4\right\rangle\left[2\left|K_{123}\right| 5\right\rangle\left[3\left|K_{123}\right| 5\right\rangle}{\left[1\left|K_{123}\right| 4\right\rangle\left[3\left|K_{123}\right| 4\right\rangle^{2}\left[3\left|K_{123}\right| 6\right\rangle t_{123}}\left(\frac{\left[2\left|K_{123}\right| 5\right\rangle\left(2 s_{12} s_{56}-\left(s_{12}+s_{56}-s_{34}\right) t_{123}\right)}{2\langle 56\rangle[12]\left[3\left|K_{123}\right| 4\right\rangle}+\langle 13\rangle[64]\right)$.

This process fixes the leading quadratic coplanar pole. Fixing the remaining linear singularity gives more terms in the amplitude. Repeating the process as before we can deduce that,

$$
\frac{1}{[3|K| 4\rangle^{2}}\left(I_{4}^{D=4}-\frac{1}{2 N_{4}}\left[\sum_{i} \alpha_{i} \gamma_{i} I_{3}^{(i)}\right]+\frac{\Delta_{4}}{\left(2 N_{4}\right)^{2}}\left[\sum_{i} \alpha_{i} \gamma_{i} I_{3}^{D=6,(i)}\right]\right) \longrightarrow \text { finite }
$$

at the coplanar singularity. The function,

$$
J_{4} \equiv\left(I_{4}^{D=4}-\frac{1}{2 N_{4}}\left[\sum_{i} \alpha_{i} \gamma_{i} I_{3}^{(i)}\right]+\frac{\Delta_{4}}{\left(2 N_{4}\right)^{2}}\left[\sum_{i} \alpha_{i} \gamma_{i} I_{3}^{D=6,(i)}\right]\right)
$$

is a combination of the $D=4$ two-mass box, $D=4$ triangle functions and $D=4$ bubble functions. If we took the box coefficients and used the $J_{4}$ functions as a basis rather than the $I_{4}$ functions, we could extend the box contributions,

$$
\sum_{i} c_{i} I_{4}^{i} \longrightarrow \sum_{i} c_{i} J_{4}^{i}
$$

to obtain an expression containing much of the three-mass triangle and bubble contributions to the amplitude. This would be an expression without $\left[3\left|K_{123}\right| 4\right\rangle$, $\left[1\left|K_{561}\right| 2\right\rangle$ or $\left[5\left|K_{345}\right| 6\right\rangle$ singularities. It may however contain linear singularities due to $\left[5\left|K_{561}\right| 2\right\rangle,\left[1\left|K_{456}\right| 4\right\rangle$ or $\left[3\left|K_{123}\right| 6\right\rangle$ vanishing.

Looking carefully at the full singularity structure, after some trial and error, we 
are led to the expression for the three-mass triangle coefficient,

$$
\begin{aligned}
& d_{3 m}^{\left[\left\{1^{-} 2^{+}\right\},\left\{3^{-} 4^{+}\right\},\left\{5^{-} 6^{+}\right\}\right]} \times(-i)= \\
&- \frac{\left[4\left|K_{345}\right| 1\right\rangle\left[5\left|K_{345}\right| 1\right\rangle\left[4\left|K_{345}\right| 6\right\rangle}{\left[5\left|K_{345}\right| 2\right\rangle\left[3\left|K_{345}\right| 6\right\rangle\left[5\left|K_{345}\right| 6\right\rangle t_{345}}\left(\frac{\left[4\left|K_{345}\right| 1\right\rangle\left(2 s_{12} s_{34}+\left(s_{56}-s_{12}-s_{34}\right) t_{345}\right)}{2\langle 12\rangle[34]\left[5\left|K_{345}\right| 6\right\rangle}+\langle 35\rangle[26]\right) \\
&- \frac{\left[6\left|K_{561}\right| 2\right\rangle\left[1\left|K_{561}\right| 3\right\rangle\left[6\left|K_{561}\right| 3\right\rangle}{\left[1\left|K_{561}\right| 2\right\rangle\left[5\left|K_{561}\right| 2\right\rangle\left[1\left|K_{561}\right| 4\right\rangle t_{561}}\left(\frac{\left[6\left|K_{561}\right| 3\right\rangle\left(2 s_{34} s_{56}+\left(s_{12}-s_{34}-s_{56}\right) t_{561}\right)}{2\langle 34\rangle[56]\left[1\left|K_{561}\right| 2\right\rangle}+\langle 51\rangle[42]\right) \\
&- \frac{\left[2\left|K_{123}\right| 4\right\rangle\left[2\left|K_{123}\right| 5\right\rangle\left[3\left|K_{123}\right| 5\right\rangle}{\left[1\left|K_{123}\right| 4\right\rangle\left[3\left|K_{123}\right| 4\right\rangle\left[3\left|K_{123}\right| 6\right\rangle t_{123}}\left(\frac{\left[2\left|K_{123}\right| 5\right\rangle\left(2 s_{12} s_{56}-\left(s_{12}+s_{56}-s_{34}\right) t_{123}\right)}{2\langle 56\rangle[12]\left[3\left|K_{123}\right| 4\right\rangle}+\langle 13\rangle[64]\right) \\
&-\left(\frac{\langle 13\rangle\langle 35\rangle[26][34]+\langle 13\rangle\langle 15\rangle[12][46]+\langle 15\rangle\langle 35\rangle[24][56]}{\Delta_{3}}\right) \times \\
&\left(\frac{\left[5\left|K_{345}\right| 1\right\rangle\left[4\left|K_{345}\right| 6\right\rangle\left(t_{345}-t_{346}\right)}{\left[5\left|K_{345}\right| 2\right\rangle\left[3\left|K_{345}\right| 6\right\rangle\left[5\left|K_{345}\right| 6\right\rangle}+\frac{\left[6\left|K_{561}\right| 2\right\rangle\left[1\left|K_{561}\right| 3\right\rangle\left(t_{561}-t_{562}\right)}{\left[1\left|K_{561}\right| 2\right\rangle\left[5\left|K_{561}\right| 2\right\rangle\left[1\left|K_{561}\right| 4\right\rangle}+\frac{\left[2\left|K_{123}\right| 4\right\rangle\left[3\left|K_{123}\right| 5\right\rangle\left(t_{123}-t_{124}\right)}{\left[1\left|K_{123}\right| 4\right\rangle\left[3\left|K_{123}\right| 4\right\rangle\left[3\left|K_{123}\right| 6\right\rangle}\right. \\
&\left.-2 \frac{\left[6\left|K_{234}\right| 2\right\rangle\left[2\left|K_{456}\right| 4\right\rangle\left[4\left|K_{612}\right| 6\right\rangle-\left[5\left|K_{345}\right| 1\right\rangle\left[1\left|K_{561}\right| 3\right\rangle\left[3\left|K_{123}\right| 5\right\rangle}{\left[5\left|K_{561}\right| 2\right\rangle\left[1\left|K_{456}\right| 4\right\rangle\left[3\left|K_{456}\right| 6\right\rangle}\right) .
\end{aligned}
$$

We have confirmed this expression by comparison with a numerical evaluation of the triple cut. This provides an alternative form for the coefficient previously obtained in ref. [9]. Our form is free of irrational expressions and has a more manifest singularity structure.

We also obtain a rational form for the other six-point three-mass triangle coefficient,

$$
\begin{aligned}
& d_{3 m}^{\left[\left\{2^{-} 3^{+}\right\},\left\{4^{-} 5^{+}\right\},\left\{6^{+} 1^{-}\right\}\right]} \times(-i)= \\
& \quad \frac{[26]\left[2\left|K_{345}\right| 4\right\rangle\left[6\left|K_{345}\right| 4\right\rangle}{[12]\left[2\left|K_{345}\right| 3\right\rangle\left[2\left|K_{345}\right| 5\right\rangle t_{345}}\left(\frac{\left[6\left|K_{345}\right| 4\right\rangle\left(2 s_{61} s_{45}+\left(s_{23}-s_{61}-s_{45}\right) t_{345}\right)}{2[61]\langle 45\rangle\left[2\left|K_{345}\right| 3\right\rangle}+\langle 12\rangle[35]\right) \\
& +\frac{\langle 51\rangle\left[3\left|K_{234}\right| 5\right\rangle\left[3\left|K_{234}\right| 1\right\rangle}{\langle 56\rangle\left[4\left|K_{234}\right| 5\right\rangle\left[2\left|K_{234}\right| 5\right\rangle t_{234}}\left(\frac{\left[3\left|K_{234}\right| 1\right\rangle\left(2 s_{23} s_{61}+\left(s_{45}-s_{23}-s_{61}\right) t_{234}\right)}{2[23]\langle 16\rangle\left[4\left|K_{234}\right| 5\right\rangle}+\langle 24\rangle[65]\right) \\
& +\frac{[13]\langle 64\rangle\left[3\left|K_{123}\right| 4\right\rangle}{[12]\langle 56\rangle\left[1\left|K_{123}\right| 6\right\rangle t_{123}}\left(\frac{\left[3\left|K_{123}\right| 4\right\rangle\left(2 s_{45} s_{23}+\left(s_{61}-s_{45}-s_{23}\right) t_{123}\right)}{2[23]\langle 45\rangle\left[1\left|K_{123}\right| 6\right\rangle}+\langle 12\rangle[65]\right) \\
& -\left(\frac{\langle 42\rangle\langle 21\rangle[32][65]+\langle 41\rangle\langle 21\rangle[61][35]+\langle 42\rangle\langle 41\rangle[45][36]}{\Delta_{3}}\right) \\
& \quad \times\left(2 \frac{[26]\langle 65\rangle[36]\langle 64\rangle-\langle 51\rangle[12]\langle 41\rangle[13]}{[12]\langle 56\rangle\left[2\left|K_{561}\right| 5\right\rangle}+\frac{[13]\langle 46\rangle\left(t_{123}-t_{623}\right)}{\langle 56\rangle[12]\left[1\left|K_{123}\right| 6\right\rangle}\right. \\
& \left.+\frac{[26]\left[2\left|K_{345}\right| 4\right\rangle\left(t_{345}-t_{245}\right)}{[12]\left[2\left|K_{345}\right| 5\right\rangle\left[2\left|K_{345}\right| 3\right\rangle}+\frac{\langle 51\rangle\left[3\left|K_{234}\right| 5\right\rangle\left(t_{234}-t_{235}\right)}{\langle 56\rangle\left[2\left|K_{234}\right| 5\right\rangle\left[4\left|K_{234}\right| 5\right\rangle}\right),
\end{aligned}
$$


which we have again confirmed by comparison with a numerical evaluation of the triple cut.

\section{Analytic evaluation of the Three-Mass Triangle Coefficients}

In this section we explore and refine some recent suggestions for using the analytic structure of triple cuts $[19,18,26]$ to evaluate the three-mass triangle coefficients.

Consider a triple cut in an amplitude where all three corners are massive,

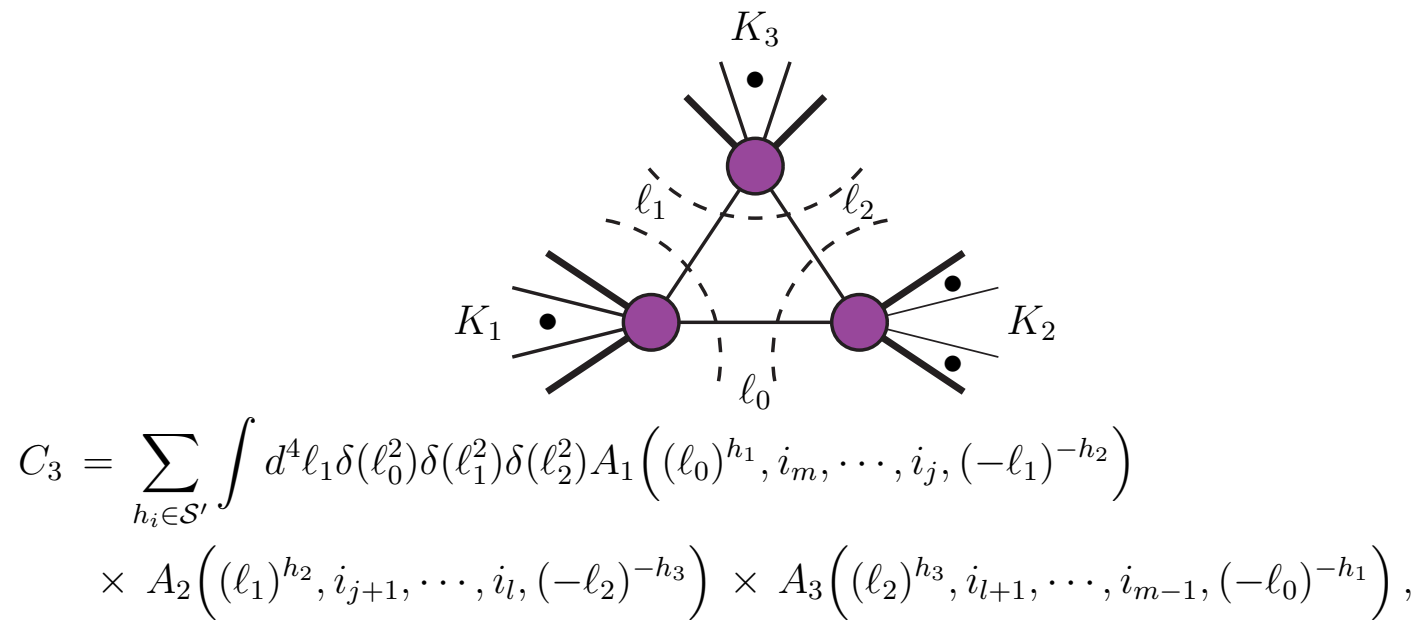

where the summation is over all possible intermediate states. As the momentum invariants, $K_{m}=k_{i_{m}}+k_{i_{m}+1}+\cdots+k_{i_{j}}$ etc, are all non-null, there exist kinematic regimes is which the integration has non-vanishing support for real loop momentum. If we expand the amplitude in terms of a basis of integral functions (1.1), the only integral functions contributing to the triple cut are box functions and the specific three-mass triangle for the cut,

$$
\begin{aligned}
C_{3} & =\sum_{i} c_{i}\left(I_{4}^{i}\right)_{\text {triple-cut }}+d_{3 m}\left(I_{3}^{3 m}\right)_{\text {triple-cut }} \\
& =\sum_{i} \frac{c_{i}}{\kappa_{i}}+d_{3 m} \frac{\pi}{2 \sqrt{-\Delta_{3}}}
\end{aligned}
$$

For the two-mass hard and three-mass boxes that arise in the six and seven-point examples we discuss, the cuts of the box integral functions are,

$$
\frac{1}{\kappa^{2 m h}}= \pm \frac{\pi}{2\left(k_{1}+k_{2}\right)^{2}\left(k_{2}+K_{3}\right)^{2}}, \frac{1}{\kappa^{3 m}}= \pm \frac{\pi}{2\left(\left(k_{1}+K_{2}\right)^{2}\left(K_{2}+K_{3}\right)^{2}-K_{2}^{2} K_{4}^{2}\right)}
$$

We will discuss the overall sign below.

Alternatively we can perform the cut integral (4.1). The triple cut is a oneparameter integral which can be calculated using algebraic methods [19]. We review 
the procedure for the general triple cut emphasising the geometric interpretation in the three-mass case as a contour integral.

The first step is to find a suitable parametrisation of the cut momenta which satisfy $l_{i}^{2}=0$ with $l_{1}=l_{0}-K_{1}$ and $l_{2}=l_{0}+K_{2}$. As $\sum K_{i}=0$, the momenta $K_{i}$ define a plane. Within this plane there exists a momentum, $a_{0}^{\mu}$, satisfying $a_{0}^{2}=$ $\left(a_{0}-K_{1}\right)^{2}=\left(a_{0}+K_{2}\right)^{2}$. Explicitly [18],

$$
a_{0}^{\mu}=\frac{K_{2}^{2}}{2} \frac{K_{1} \cdot K_{2}+K_{1}^{2}}{K_{1}^{2} K_{2}^{2}-\left(K_{1} \cdot K_{2}\right)^{2}} K_{1}^{\mu}-\frac{K_{1}^{2}}{2} \frac{K_{1} \cdot K_{2}+K_{2}^{2}}{K_{1}^{2} K_{2}^{2}-\left(K_{1} \cdot K_{2}\right)^{2}} K_{2}^{\mu} .
$$

In the three-mass case, $\left|a_{0}\right| \neq 0$, the cut momenta are real and for $a_{0}$ time-like can be parameterised in the form,

$$
\ell_{0}^{\mu}=a_{0}^{\mu}+\rho\left(\cos \theta m^{\mu}+\sin \theta n^{\mu}\right)
$$

where $\rho=\sqrt{-a_{0}^{2}}$ and $0 \leq \theta \leq 2 \pi$. The vectors $m$ and $n$ are mutually orthogonal unit vectors which are orthogonal to the $\left(K_{1}, K_{2}\right)$ plane; $(m \cdot n)=\left(m \cdot K_{i}\right)=$ $\left(n \cdot K_{i}\right)=0$. For $a_{0}$ space-like, a hyperbolic parametrisation can be used. If we now define the complex null momenta, $r=\frac{\rho}{2}(m+i n)$ and $\bar{r}=\frac{\rho}{2}(m-i n)$, we recover the parametrisation used in $[18,19]^{2}$,

$$
\ell_{i}^{\mu}=t \bar{r}^{\mu}+\frac{1}{t} r^{\mu}+a_{i}^{\mu}
$$

where $t=\mathrm{e}^{i \theta}, a_{1}=a_{0}-K_{1}$ and $a_{2}=a_{0}+K_{2}$.

With this parametrisation it is clear that the cut integration becomes a contour integration over the complex variable $t$ with the contour being the unit circle. The integral then becomes,

$$
\int d^{4} l \prod_{i} \delta\left(\ell_{i}^{2}\right)(\bullet) \longrightarrow \int d t J_{t}(\bullet)
$$

where $J_{t}=1 /(t \sqrt{\Delta})$ is the Jacobian and $\Delta=16 \Delta_{3}$. Regarding $t$ as complex allows the integral to be performed analytically using contour methods. In the three-mass case the contour is well specified.

Parameterising the loop momenta according to (4.6) the product of tree amplitudes $A_{1} A_{2} A_{3}$ is a rational function of $t$. This rational function will have simple poles at $t=t_{i} \neq 0$ and, possibly, non-simple poles at $t=0$. Poles in this product at $t=t_{i} \neq 0$ arise when one of the tree amplitudes factorises and some momentum, $\hat{P}(t)$, becomes null:

$$
A \underset{\hat{P}^{2} \rightarrow 0}{\longrightarrow} \hat{A}_{L} \frac{1}{\hat{P}(t)^{2}} \hat{A}_{R}
$$

\footnotetext{
${ }^{2}$ The $t$ in our parametrisation and that in [19] are related by a scaling
} 
where $\hat{A}_{L}$ and $\hat{A}_{R}$ are tree amplitudes evaluated at the momenta where $\hat{P}^{2}$ vanishes. In general $\hat{P}^{2}=0$ gives two poles. For the six and seven-point examples we discuss, one of these poles gives the box contribution while the other gives no contribution. In these examples each box has at least one massless corner and we have $\hat{P}=l \pm a$, where $a$ is the external momentum of a massless corner. Poles arise when either $\langle l a\rangle=0$ or $[l a]=0$. The original tree amplitudes will only contain one of these poles, so only one of the poles can contribute to the triple cut. If the appropriate pole is inside the contour of integration, the contribution to the triple cut is of the form,

$$
\left.2 \pi i \operatorname{Res}\left(\frac{A_{1} A_{2} \hat{A}_{3 L} \hat{A}_{3 R}}{t \sqrt{\Delta} \hat{P}^{2}}\right)\right|_{t=t_{i}}
$$

By comparison with the quadruple cut procedure, we see that the product of onshell tree amplitudes reproduces the box coefficient up to a factor of 2 . It is useful to compare the rest of this expression to the triple cut of the corresponding scalar box,

$$
\int d t J_{t} \frac{1}{\left(l_{0}-P\right)^{2}}=\int \frac{d t}{t \sqrt{\Delta}} \frac{1}{\hat{P}^{2}} .
$$

This has poles in identical positions, but both could in principle contribute. Denoting the two $t$-values for which $\left(l_{0}-P\right)^{2}$ vanishes by $t_{ \pm}$, we have,

$$
\frac{1}{t\left(\ell_{0}-P\right)^{2}}=\frac{-1}{(2 \bar{r} \cdot P)\left(t-t_{+}\right)\left(t-t_{-}\right)}=\frac{-1}{(2 \bar{r} \cdot P)\left(t_{+}-t_{-}\right)}\left(\frac{1}{t-t_{+}}-\frac{1}{t-t_{-}}\right) \text {. }
$$

The two poles thus have equal but opposite residues. In the three-mass case, $t_{+}$and $t_{-}$are the roots the quadratic equation,

$$
2 \bar{r} \cdot P t^{2}+\left(2 a_{0} \cdot P-P^{2}\right) t+2 r \cdot P=0
$$

so the product of the roots is,

$$
t_{+} t_{-}=\frac{r \cdot P}{\bar{r} \cdot P} \rightarrow\left|t_{+} t_{-}\right|=1
$$

One pole is always inside the unit circle and one is outside. Thus the triple cut of the box function always gives a contribution, but the sign depends on the kinematic point. In contrast, the original triple cut integral only receives contributions if the appropriate pole is inside the contour of integration.

In general $\left(A_{1} A_{2} A_{3}\right) / t$ can also have a pole at $t=0$ and we denote the residue of this pole by $\rho_{0}$. Using both approaches to evaluate the triple cut integral we then have,

$$
C_{3}=\sum_{i} 2 \Theta\left(1-\left|t_{i}\right|\right) \frac{c_{i}}{\tau_{i}}+\frac{2 \pi \rho_{0}}{\sqrt{-\Delta_{3}}}=\sum_{i}-(-1)^{\Theta\left(1-\left|t_{i}\right|\right)} \frac{c_{i}}{\tau_{i}}+d_{3 m} \frac{\pi}{2 \sqrt{-\Delta_{3}}}
$$


where, $\tau_{i}=-(-1)^{\Theta\left(1-\left|t_{i}\right|\right)} \kappa_{i}$. We can rearrange this to give an expression free from $\Theta$ functions,

$$
\frac{2 \pi \rho_{0}}{\sqrt{-\Delta_{3}}}=\sum_{i}-\frac{c_{i}}{\tau_{i}}+d_{3 m} \frac{\pi}{2 \sqrt{-\Delta_{3}}} \equiv \mathcal{S} \equiv \mathcal{S}^{\text {box }}+\mathcal{S}^{\text {triangle }}
$$

which relates $\rho_{0}$ to a specific sum of box and triangle contributions. The box contributions are readily calculated either by quadruple cuts or by using the fact that they are half of the $t \neq 0$ residues. The latter approach provides a realisation of the quadruple cut procedure that is amenable to automation [19]. The three-mass triangle contribution to $\rho_{0}$ can thus readily be identified. A slightly different formulation involving integration over two different regions (corresponding to the interior and exterior of the unit circle in this case) was presented in [19].

For any $n$-point NMHV amplitude the three tree amplitudes in the triple-cut of a three-mass triangle are of MHV type. When we parameterise the integral by $t$, each tree amplitude has a $t^{-1}$ factor since each contains a $\left\langle l_{i} l_{i+1}\right\rangle^{-1}$ factor and, for example,

$$
\begin{aligned}
\left\langle l_{0} l_{1}\right\rangle=\frac{\left[r\left|l_{0} l_{1}\right| r\right]}{\left[r l_{0}\right]\left[l_{1} r\right]} & =\frac{\left[r\left|\left(t \bar{r}+t^{-1} r+a_{0}\right)\left(t \bar{r}+t^{-1} r+a_{0}-K_{1}\right)\right| r\right]}{\left[r l_{0}\right]\left[l_{1} r\right]} \\
& =\frac{\left[r\left|t \bar{r}\left(a_{0}-K_{1}\right)+t a_{0} \bar{r}+a_{0}\left(a_{0}-K_{1}\right)\right| r\right]}{\left[r l_{0}\right]\left[l_{1} r\right]} \\
& =t \frac{\left[r\left|\bar{r}\left(a_{0}-K_{1}\right)+a_{0} \bar{r}\right| r\right]}{\left[r l_{0}\right]\left[l_{1} r\right]}=t \frac{-\left[r\left|\bar{r} K_{1}\right| r\right]}{\left[r l_{0}\right]\left[l_{1} r\right]}
\end{aligned}
$$

using the orthogonality properties of $r, \bar{r}, a_{0}$ and $K_{1}$. The $\left[r l_{i}\right]$ factors cancel overall as the product of tree amplitudes has no spinor weight in $l_{i}$. Thus, for each particle circulating in the loop, the integrand has a $t^{-3}$ factor and $\rho_{0}$ must be extracted by expanding around this triple pole.

For the $\mathcal{N}=1$ coefficients we present, summing over the particle types leads to cancellations. Relative to the case of a scalar in the loop, the $\mathcal{N}=1$ multiplet has an overall factor. Denoting the three negative helicity external legs by $m_{i}$, this factor is,

$$
\frac{\left(\left\langle l_{0} m_{1}\right\rangle\left\langle l_{1} m_{2}\right\rangle\left\langle l_{2} m_{3}\right\rangle-\left\langle l_{2} m_{1}\right\rangle\left\langle l_{0} m_{2}\right\rangle\left\langle l_{1} m_{3}\right\rangle\right)^{2}}{\left\langle l_{0} m_{1}\right\rangle\left\langle l_{1} m_{2}\right\rangle\left\langle l_{2} m_{3}\right\rangle\left\langle l_{2} m_{1}\right\rangle\left\langle l_{0} m_{2}\right\rangle\left\langle l_{1} m_{3}\right\rangle} .
$$

Cancellations in the numerator give this expression an overall factor of $t^{2}$ implying that the full $\mathcal{N}=1$ integrand diverges as $t^{-1}$. $\rho_{0}$ can then be extracted by taking a derivative:

$$
\rho_{0} \sim \frac{d}{d t}\left(t\left(A_{1} A_{2} A_{3}\right)\right)_{t \rightarrow 0} .
$$

For $\mathcal{N}=4$ the overall factor is that of $\mathcal{N}=1$ squared and thus introduces a $t^{4}$ factor. For these amplitudes it is thus trivial to see that $\rho_{0}=0$ and there are no three-mass 
triangles present in the expansion. This argument easily extends to show that there are no three-mass triangles present in $\mathcal{N}=8$ supergravity [27].

This approach can also be used to generate relationships between the triple cuts of amplitudes with different helicity configurations. In the next section we discuss this in the context of seven-point amplitudes.

\section{Seven-Point Results}

In ref. [20] it was shown how on-shell recursion techniques could be applied to integral coefficients by utilising complex momentum shifts similar to those used for tree level gluon amplitudes [28]. Under certain conditions the integral coefficient within an

$n+1$ point amplitude can be obtained from the lower point amplitudes via the factorisations,

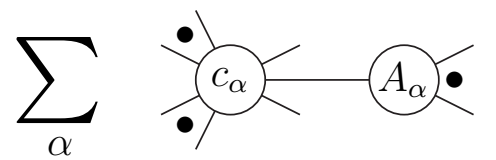

The six-point three-mass coefficients given in section 4 are in a form which can be used for this technique. The independent seven-point NMHV amplitudes are,

$$
\begin{array}{ll}
A\left(1^{-}, 2^{-}, 3^{-}, 4^{+}, 5^{+}, 6^{+}, 7^{+}\right), & A\left(1^{-}, 2^{-}, 3^{+}, 4^{-}, 5^{+}, 6^{+}, 7^{+}\right), \\
A\left(1^{-}, 2^{-}, 3^{+}, 4^{+}, 5^{-}, 6^{+}, 7^{+}\right), & A\left(1^{-}, 2^{+}, 3^{-}, 4^{+}, 5^{-}, 6^{+}, 7^{+}\right) .
\end{array}
$$

The three-mass triangles appearing in these amplitudes are,

$$
\begin{aligned}
&\left.A\left(1^{-}, 2^{-}, 3^{-}, 4^{+}, 5^{+}, 6^{+}, 7^{+}\right)\right|_{3 m}: \text { none }, \\
&\left.A\left(1^{-}, 2^{-}, 3^{+}, 4^{-}, 5^{+}, 6^{+}, 7^{+}\right)\right|_{3 m}: I^{\left[\left\{2^{-} 3^{+}\right\},\left\{4^{-} 5^{+}\right\},\left\{6^{+} 7^{+} 1^{-}\right\}\right]}, I^{\left[\left\{2^{-} 3^{+}\right\},\left\{4^{-} 5^{+} 6^{+}\right\},\left\{7^{+} 1^{-}\right\}\right]}, \\
&\left.A\left(1^{-}, 2^{-}, 3^{+}, 4^{+}, 5^{-}, 6^{+}, 7^{+}\right)\right|_{3 m}: I^{\left[\left\{2^{-} 3^{+} 4^{+}\right\},\left\{5^{-} 6^{+}\right\},\left\{7^{+} 1^{-}\right\}\right]}, I^{\left[\left\{2^{-} 3^{+}\right\},\left\{4^{+} 5^{-}\right\},\left\{6^{+} 7^{+} 1^{-}\right\}\right]}, \\
& I^{\left[\left\{2^{-} 3^{+}\right\},\left\{4^{+} 5^{-} 6^{+}\right\},\left\{7^{+} 1^{-}\right\}\right]}, \\
&\left.A\left(1^{-}, 2^{+}, 3^{-}, 4^{+}, 5^{-}, 6^{+}, 7^{+}\right)\right|_{3 m}: I^{\left[\left\{1^{-} 2^{+}\right\},\left\{3^{-} 4^{+}\right\},\left\{5^{-} 6^{+} 7^{+}\right\}\right]}, I^{\left[\left\{7^{+} 1^{-}\right\},\left\{2^{+} 3^{-}\right\},\left\{4^{+} 5^{-} 6^{+}\right\}\right]}, \\
& I^{\left[\left\{7^{+} 1^{-}\right\},\left\{2^{+} 3^{-} 4^{+}\right\},\left\{5^{-} 6^{+}\right\}\right]}, I^{\left[\left\{6^{+} 7^{+} 1^{-}\right\},\left\{2^{+} 3^{-}\right\},\left\{4^{+} 5^{-}\right\}\right]}, \\
& I^{\left[\left\{7^{+} 1^{-} 2^{+}\right\},\left\{3^{-} 4^{+}\right\},\left\{5^{-} 6^{+}\right\}\right]} .
\end{aligned}
$$

The coefficients of these are not all independent since,

$$
\begin{aligned}
& d_{3 m}^{\left[\left\{2^{-} 3^{+} 4^{+}\right\},\left\{5^{-} 6^{+}\right\},\left\{7^{+} 1^{-}\right\}\right]}=\left.d_{3 m}^{\left[\left\{2^{-} 3^{+}\right\},\left\{4^{+} 5^{-}\right\},\left\{6^{+} 7^{+} 1^{-}\right\}\right]}\right|_{1234567 \leftrightarrow 2176543}, \\
& d_{3 m}^{\left[\left\{1^{-} 2^{+}\right\},\left\{3^{-} 4^{+}\right\},\left\{5^{-} 6^{+} 7^{+}\right\}\right]}=\left.d_{3 m}^{\left[\left\{6^{+} 7^{+} 1^{-}\right\},\left\{2^{+} 3^{-}\right\},\left\{4^{+} 5^{-}\right\}\right]}\right|_{1234567 \leftrightarrow 5432176}, \\
& d_{3 m}^{\left[\left\{7^{+} 1^{-}\right\},\left\{2^{+} 3^{-}\right\},\left\{4^{+} 5^{-} 6^{+}\right\}\right]}=\left.d_{3 m}^{\left[\left\{7^{+} 1^{-} 2^{+}\right\},\left\{3^{-} 4^{+}\right\},\left\{5^{-} 6^{+}\right\}\right]}\right|_{1234567 \leftrightarrow 5432176},
\end{aligned}
$$

leaving seven independent coefficients. 
A recursive approach is particularly simple for the coefficients where the helicities are "split": that is the negative and positive helicities on a corner are clustered, $\{-\cdots--++\cdots+\}$. For the seven point amplitudes this corresponds to looking at coefficients where the corner with three external legs has helicity structure -++ or ++- rather than +-+ .

If we consider the three-mass triangle $I_{3}^{3 m}(\{23\},\{45\},\{671\})$ and apply the shift,

$$
\lambda_{7} \rightarrow \hat{\lambda}_{7}=\lambda_{7}+z \lambda_{1}, \quad \bar{\lambda}_{1} \rightarrow \bar{\lambda}_{1}-z \bar{\lambda}_{7}
$$

then the seven point coefficient is related to the six-point by,

$$
\begin{aligned}
d_{3 m}^{\left[\left\{2^{-} 3^{+}\right\},\left\{4^{-} 5^{+}\right\},\left\{6^{+} 7^{+} 1^{-}\right\}\right]} & =d_{3 m}^{\left[\left\{2^{-} 3^{+}\right\},\left\{4^{-} 5^{+}\right\},\left\{\hat{K}_{67}^{+} \hat{1}^{-}\right\}\right]} \frac{i}{s_{67}} A\left(\hat{7}^{+}, 6^{+},\left(-\hat{K}_{67}\right)^{-}\right) \\
& =d_{3 m}^{\left[\left\{2^{-} 3^{+}\right\},\left\{4^{-} 5^{+}\right\},\left\{\hat{K}_{67}^{+} \hat{1}^{-}\right\}\right]} \frac{i}{s_{67}} \frac{i[76]^{3}}{\left[6 \hat{K}_{67}\right]\left[\hat{K}_{67} 7\right]} .
\end{aligned}
$$

where the "hatted" objects are the shifted values and $z$ takes the value for which $\hat{K}_{67}$ is null. After evaluating the shifted amplitudes we have,

$$
\begin{aligned}
& d_{3 m}^{\left[\left\{2^{-} 3^{+}\right\},\left\{4^{-} 5^{+}\right\},\left\{6^{+} 7^{+} 1^{-}\right\}\right]} \times(-i)(\langle 17\rangle\langle 76\rangle\langle 61\rangle)= \\
& \frac{\left[2\left|K_{A}\right| 4\right\rangle\left\langle 1\left|K_{B} K_{A}\right| 4\right\rangle\left[2\left|K_{A}\right| 1\right\rangle\langle 61\rangle}{\left[2\left|K_{A}\right| 3\right\rangle\left[2\left|K_{A}\right| 5\right\rangle\left[2\left|K_{A}\right| 6\right\rangle t_{A}}\left(\frac{t_{A}\left(s_{23}-t_{B}-s_{45}\right)+2 t_{B} s_{45}}{2\left[2\left|K_{A}\right| 3\right\rangle} \frac{\left\langle 1\left|K_{B} K_{A}\right| 4\right\rangle}{t_{B}\langle 54\rangle}+\langle 21\rangle[35]\right) \\
& -\frac{\langle 51\rangle\langle 16\rangle\left[3\left|K_{C}\right| 5\right\rangle\left[3\left|K_{C}\right| 1\right\rangle}{\langle 65\rangle\left[4\left|K_{C}\right| 5\right\rangle\left[2\left|K_{C}\right| 5\right\rangle t_{C}}\left(\frac{t_{C}\left(s_{45}-s_{23}-t_{B}\right)+2 s_{23} t_{B}}{2\left[4\left|K_{C}\right| 5\right\rangle} \frac{\left[3\left|K_{C}\right| 1\right\rangle}{[23]}+\left[5\left|K_{C}\right| 1\right\rangle\langle 42\rangle\right) \\
& -\frac{\left(\left[3\left|K_{D}\right| 4\right\rangle\langle 61\rangle+[37]\langle 14\rangle\langle 67\rangle\right)\left[3\left|K_{B}\right| 6\right\rangle\langle 64\rangle\langle 16\rangle^{2}}{\left\langle 6\left|K_{B} K_{23}\right| 6\right\rangle\left[2\left|K_{B}\right| 6\right\rangle\langle 65\rangle \hat{t}_{D}} \times \\
& \left(\frac{\hat{t}_{D}\left(t_{B}-s_{23}-s_{45}\right)+2 s_{45} s_{23}}{2\left\langle 6\left|K_{71} K_{23}\right| 6\right\rangle} \frac{\left(\left[3\left|K_{D}\right| 4\right\rangle\langle 61\rangle+[37]\langle 14\rangle\langle 67\rangle\right)}{[32]\langle 54\rangle}+\frac{\langle 21\rangle\left[5\left|K_{B}\right| 1\right\rangle}{\langle 61\rangle}\right) \\
& +\left(\frac{\langle 42\rangle\langle 41\rangle[45]\left[3\left|K_{B}\right| 1\right\rangle-\langle 42\rangle\langle 21\rangle[32]\left[5\left|K_{B}\right| 1\right\rangle-\langle 41\rangle\langle 21\rangle t_{B}[35]}{\Delta_{3}}\right) \times \\
& \left(2 \frac{\left[2\left|K_{B}\right| 1\right\rangle\langle 65\rangle\left[3\left|K_{B}\right| 1\right\rangle\langle 64\rangle-\langle 51\rangle\left[2\left|K_{B}\right| 6\right\rangle\langle 41\rangle\left[3\left|K_{B}\right| 6\right\rangle}{\langle 65\rangle\left[2\left|K_{B}\right| 6\right\rangle\left[2\left|K_{B}\right| 5\right\rangle}-\frac{\langle 51\rangle\langle 16\rangle\left[3\left|K_{C}\right| 5\right\rangle\left(t_{C}-t_{235}\right)}{\langle 65\rangle\left[2\left|K_{C}\right| 5\right\rangle\left[4\left|K_{C}\right| 5\right\rangle}\right. \\
& \left.+\frac{\left[2\left|K_{B}\right| 1\right\rangle\langle 16\rangle\left[2\left|K_{A}\right| 4\right\rangle\left(t_{A}-t_{245}\right)}{\left[2\left|K_{B}\right| 6\right\rangle\left[2\left|K_{A}\right| 5\right\rangle\left[2\left|K_{A}\right| 3\right\rangle}-\frac{\left[3\left|K_{B}\right| 6\right\rangle\langle 64\rangle\langle 16\rangle^{2}\left(2 \hat{t}_{D}-s_{45}+t_{B}-s_{23}\right)}{\langle 65\rangle\left[2\left|K_{B}\right| 6\right\rangle\left\langle 6\left|K_{B} K_{23}\right| 6\right\rangle}\right)
\end{aligned}
$$

where $K_{A}=K_{345}, K_{B}=K_{671}, K_{C}=K_{234}, K_{D}=K_{123}$ and $\hat{t}_{123}=-\left\langle 6\left|K_{45} K_{23}\right| 1\right\rangle /\langle 61\rangle$. 
Similarly,

$$
\begin{aligned}
d_{3 m}^{\left[\left\{2^{-} 3^{+} 4^{+}\right\},\left\{5^{-} 6^{+}\right\},\left\{7^{+} 1^{-}\right\}\right]}= & d_{3 m}^{\left[\left\{\hat{2}^{-} \hat{K}_{34}\right\},\left\{5^{-} 6^{+}\right\},\left\{7^{+} 1^{-}\right\}\right]} \times \frac{i}{s_{34}} \times A_{3}\left(3^{+}, 4^{+},\left(-\hat{K}_{34}\right)^{-}\right) \\
& : \hat{\lambda}_{3}=\lambda_{3}+z \lambda_{2}, \hat{\bar{\lambda}}_{2}=\bar{\lambda}_{2}-z \bar{\lambda}_{1}, z=-\langle 43\rangle /\langle 42\rangle, \\
d_{3 m}^{\left[\left\{6^{+} 7^{+} 1^{-}\right\},\left\{2^{+} 3^{-}\right\},\left\{4^{+} 5^{-}\right\}\right]}= & d_{3 m}^{\left[\left\{\hat{K}_{67}^{+} \hat{1}^{-}\right\},\left\{2^{+} 3^{-}\right\},\left\{4^{+} 5^{-}\right\}\right]} \times \frac{i}{s_{67}} \times A_{3}\left(6^{+}, 7^{+},\left(-\hat{K}_{67}\right)^{-}\right) \\
& : \hat{\lambda}_{7}=\lambda_{7}+z \lambda_{1}, \hat{\bar{\lambda}}_{1}=\bar{\lambda}_{1}-z \bar{\lambda}_{7}, z=-\langle 67\rangle /\langle 61\rangle, \\
d_{3 m}^{\left[\left\{2^{-} 3^{+}\right\},\left\{4^{-} 5^{+} 6^{+}\right\},\left\{7^{+} 1^{-}\right\}\right]}= & d_{3 m}^{\left[\left\{2^{-} 3^{+}\right\},\left\{4^{-} \hat{K}_{56}^{+}\right\},\left\{7^{+} 1^{-}\right\}\right]} \times \frac{i}{s_{56}} \times A_{3}\left(5^{+}, 6^{+},\left(-\hat{K}_{56}\right)^{-}\right) \\
& : \hat{\lambda}_{5}=\lambda_{5}+z \lambda_{4}, \hat{\bar{\lambda}}_{4}=\bar{\lambda}_{4}-z \bar{\lambda}_{5}, z=-\langle 65\rangle /\langle 64\rangle .
\end{aligned}
$$

We can express the remaining three coefficients in terms of these split helicity coefficients (together with box coefficients) by using their analytic structure. The three possibilities for a corner with two positive helicity external legs and a single negative helicity external leg are,

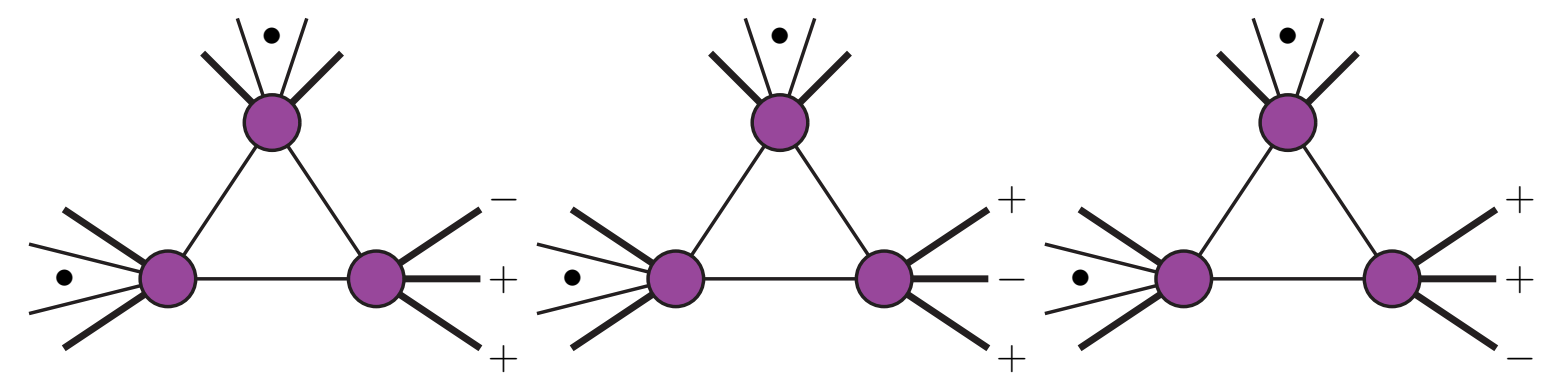

We denote these amplitudes from left to right by $A_{L}=A\left((a \ldots b)(c \ldots d)\left(e^{-}, f^{+}, g^{+}\right)\right)$, $A_{M}=A\left((a \ldots b)(c \ldots d)\left(f^{+}, e^{-}, g^{+}\right)\right)$and $A_{R}=A\left((a \ldots b)(c \ldots d)\left(f^{+}, g^{+}, e^{-}\right)\right)$. The two split helicity configurations can be obtained from the six-point amplitudes by recursion.

In the cut the five-point corner is an MHV tree amplitude, so we can write the integrands, $X=A_{1} A_{2} A_{3}$, of the respective triple cut integrals as,

$$
X_{M}=X_{L} f_{L}, \quad X_{R}=X_{L} f_{L} f_{R},
$$

where $f_{L}$ and $f_{R}$ are simple factors. From (4.18) we see that the quantity of interest is $\left.\frac{d}{d t}\left(t X_{M}\right)\right|_{t=0}$, while we know $\left.\frac{d}{d t}\left(t X_{L}\right)\right|_{t=0}$ and $\left.\frac{d}{d t}\left(t X_{R}\right)\right|_{t=0}$. Using the relations above,

$$
\begin{array}{r}
\left.\frac{d}{d t}\left(t X_{M}\right)\right|_{t=0}=\left.\left.\frac{d}{d t}\left(t X_{L}\right)\right|_{t=0} f_{L}\right|_{t=0}+\left.\left.\left(t X_{L}\right)\right|_{t=0} \frac{d\left(f_{L}\right)}{d t}\right|_{t=0}, \\
\left.\frac{d}{d t}\left(t X_{R}\right)\right|_{t=0}=\left.\left.\frac{d}{d t}\left(t X_{L}\right)\right|_{t=0}\left(f_{L} f_{R}\right)\right|_{t=0}+\left.\left.\left(t X_{L}\right)\right|_{t=0} \frac{d\left(f_{L} f_{R}\right)}{d t}\right|_{t=0} .
\end{array}
$$


Eliminating $\left.\left(t X_{L}\right)\right|_{t=0}$ and dropping the explicit $\left.\right|_{t=0}$ 's for clarity, we have,

$$
\mathcal{S}_{M}=\frac{\mathcal{S}_{L} f_{L}^{2} f_{R}^{\prime}+\mathcal{S}_{R} f_{L}^{\prime}}{f_{L}^{\prime} f_{R}+f_{L} f_{R}^{\prime}} \equiv \alpha_{L} \mathcal{S}_{L}+\alpha_{R} \mathcal{S}_{R}
$$

where $\mathcal{S}$ contains the three-mass triangle and box contributions.

In the particular example here, the five-point denominators and $f_{L}$ and $f_{R}$ are given by,

$$
\begin{aligned}
A_{L}: & \frac{1}{\left\langle l_{2} e\right\rangle\langle e f\rangle\langle f g\rangle\left\langle g l_{0}\right\rangle\left\langle l_{0} l_{2}\right\rangle}, \quad f_{L}=-\frac{\left\langle l_{2} e\right\rangle\langle f g\rangle}{\left\langle l_{2} f\right\rangle\langle e g\rangle}, \\
A_{M}: & \frac{1}{\left\langle l_{2} f\right\rangle\langle f e\rangle\langle e g\rangle\left\langle g l_{0}\right\rangle\left\langle l_{0} l_{2}\right\rangle}, \\
A_{R}: & \frac{1}{\left\langle l_{2} f\right\rangle\langle f g\rangle\langle g e\rangle\left\langle e l_{0}\right\rangle\left\langle l_{0} l_{2}\right\rangle}, \quad f_{R}=-\frac{\left\langle g l_{0}\right\rangle\langle f e\rangle}{\left\langle e l_{0}\right\rangle\langle f g\rangle},
\end{aligned}
$$

which give,

$$
\alpha_{L}^{-1}=-1-\frac{\langle f e\rangle\left[r\left|K_{2}\right| g\right\rangle}{\langle g f\rangle\left[r\left|a_{0}+K_{2}\right| e\right\rangle}, \quad \alpha_{R}^{-1}=-1-\frac{\langle e g\rangle\left[r\left|K_{2}\right| f\right\rangle}{\langle f g\rangle\left[r\left|a_{0}\right| e\right\rangle} .
$$

When one constructs $\bar{\lambda}_{r}$, terms containing $\sqrt{-\Delta_{3}}$ appear. In the language of [18] these arise when constructing null vectors in the $\left(K_{1}, K_{2}\right)$ plane. $\alpha_{L}$ and $\alpha_{R}$ thus have terms containing $\sqrt{-\Delta_{3}}$ and these conspire with the $\sqrt{-\Delta_{3}}$ factors entering (5.11) via the cuts of the triangle functions to give rational triangle coefficients. Expressing $\alpha_{L}$ and $\alpha_{R}$ in forms where the $\sqrt{-\Delta_{3}}$ factors are explicit,

$$
\alpha_{L}=\alpha_{L}^{R a t}+\alpha_{L}^{I r r} \sqrt{-\Delta_{3}}, \quad \alpha_{R}=\alpha_{R}^{R a t}+\alpha_{R}^{I r r} \sqrt{-\Delta_{3}},
$$

we then have,

$$
\begin{aligned}
\mathcal{S}_{M}^{\text {box }} & =\alpha_{L}^{\text {Rat }} \mathcal{S}_{L}^{\text {box }}+\alpha_{R}^{R a t} \mathcal{S}_{R}^{\text {box }}+\frac{\pi}{2}\left(\alpha_{L}^{I r r} d_{L}^{3 m}+\alpha_{R}^{I r r} d_{R}^{3 m}\right) \\
d_{M}^{3 m} & =\alpha_{L}^{R a t} d_{L}^{3 m}+\alpha_{R}^{R a t} d_{R}^{3 m}-\frac{2 \Delta_{3}}{\pi}\left(\alpha_{L}^{I r r} \mathcal{S}_{L}^{\text {box }}+\alpha_{R}^{I r r} \mathcal{S}_{R}^{\text {box }}\right) .
\end{aligned}
$$

These expressions can be used to obtain the coefficients: $d_{3 m}^{\left[\left\{2^{-} 3^{+}\right\},\left\{4^{+} 5^{-} 6^{+}\right\},\left\{7^{+} 1^{-}\right\}\right]}$, $d_{3 m}^{\left[\left\{7^{+} 1^{-}\right\},\left\{2^{+} 3^{-} 4^{+}\right\},\left\{5^{-} 6^{+}\right\}\right]}$and $d_{3 m}^{\left[\left\{7^{+} 1^{-} 2^{+}\right\},\left\{3^{-} 4^{+}\right\},\left\{5^{-} 6^{+}\right\}\right]}$, which can be confirmed numerically.

\section{NMHV Three-Mass Triangles From Recursion}

In this section we discuss how the recursive methods of [20] may be used to generate the three-mass triangle coefficients for the $n$-point $\mathrm{NMHV} \mathcal{N}=1$ amplitudes. 
The first step is to shift two adjacent external legs, $i$ and $j$, in one corner of the triangle according to,

$$
\lambda_{i} \longrightarrow \lambda_{i}+z \lambda_{j}, \quad \bar{\lambda}_{j} \longrightarrow \bar{\lambda}_{j}-z \bar{\lambda}_{i} .
$$

We will explicitly describe the case where legs $i$ and $j$ have one specific ordering, the case with the other ordering is directly analogous. In general the tree amplitude will factorise as,
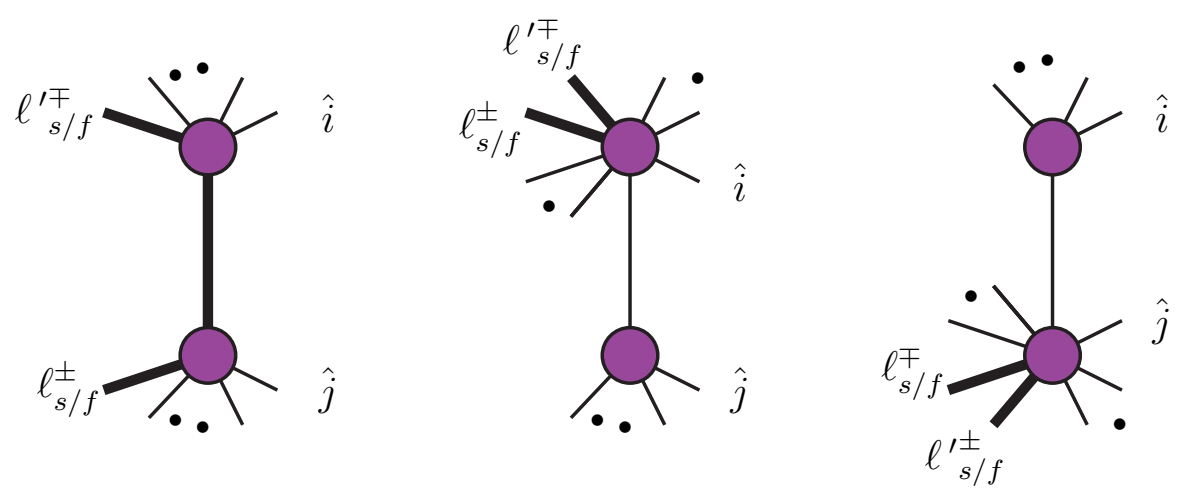

where we have separated the diagrams depending on the position of the loop momentum legs. Thick lines indicate propagators with loop momentum dependence. Diagrams of the second and third type give contributions that are readily expressed in terms of lower point triangle coefficients. These contributions will reproduce the $n$-point coefficient providing the first diagram is absent and the original shifted tree amplitude vanishes as $|z| \rightarrow \infty$. Sufficient criteria for a valid recursion are thus [20]:

1. The shifted tree amplitude vanishes as $|z| \rightarrow \infty$.

2. All loop momentum dependent kinematic poles are unmodified by the shift.

For the $\mathcal{N}=1$ multiplet the coefficients of NMHV three-mass triangles will vanish unless each corner contains exactly one negative helicity external leg. The tree amplitudes at each corner are therefore MHV amplitudes and the first condition is satisfied if leg $i$ is of positive helicity. If in addition we take leg $j$ to have negative helicity, the problematic factorisation has the form,

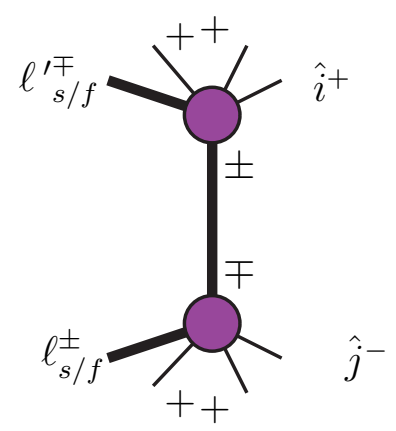


The lower amplitude in this factorisation is always MHV and hence non-vanishing. However the upper amplitude only has a single negative helicity leg and so vanishes unless it is has precisely three legs. The problematic factorisation is therefore absent if there is at least one more positive helicity external leg adjacent to leg $i$. By the same argument we see that the other factorisations must also involve a three-point $\overline{\mathrm{MHV}}$ vertex. Only one of these three-point $\overline{\mathrm{MHV}}$ vertices is non-vanishing under (6.1), so there is a single contribution:

$$
\begin{aligned}
& \left.d_{3 m}^{\left[\left\{C_{1}\right\},\left\{C_{2}\right\},\left\{a \cdots, i-2^{+}, i-1^{+}, i^{+}, j^{-}, j+1^{+}, \cdots b\right\}\right]}\right] \\
& \quad=\quad d_{3 m}^{\left[\left\{C_{1}\right\},\left\{C_{2}\right\},\left\{a \cdots, i-2^{+}, \hat{K}^{+}, j^{-}, j+1^{+}, \cdots b\right\}\right]} \frac{i}{s_{i, i-1}} A\left((-\hat{K})^{-}, i-1^{+}, \hat{i}^{+}\right) .
\end{aligned}
$$

This procedure gives the $n$-point coefficient in terms of the $n$-1-point coefficient with one less positive helicity leg in the cluster containing leg $i$. Repeated applications of this procedure will produce an expression for the original $n$-point coefficient in terms of a coefficient with either one or no positive helicity legs on either side of the negative helicity leg at each corner. Thus we need three-mass triangle coefficients for those functions where each corner takes one of the three forms below:
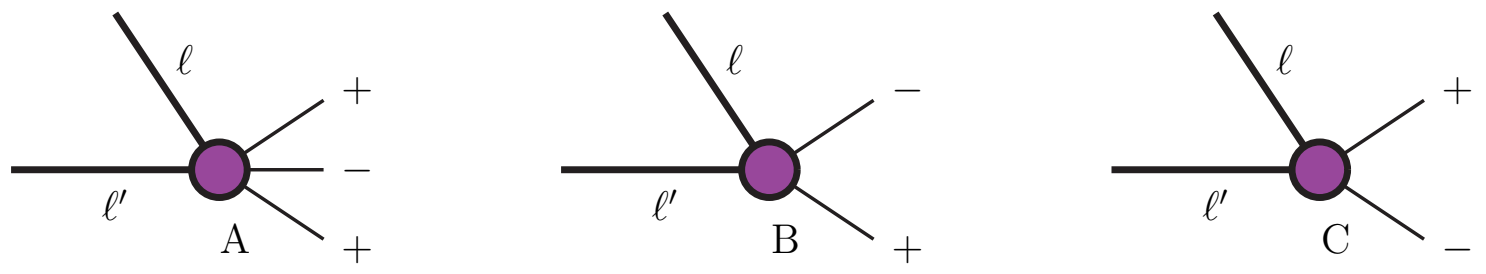

We have described in the previous sections how to generate coefficients for the seven-point functions with a single corner of type A. As both the recursive and helicity reordering techniques address one corner of the triangle at a time, they can be applied to the other two corners of these seven-point amplitudes to produce the eight and nine-point amplitudes containing two and three corners of type A respectively. This procedure will produce all of the coefficients that are required for the recursive generation of any $n$-point NMHV $\mathcal{N}=1$ three-mass triangle coefficient.

\section{Conclusions}

We have discussed a range of techniques that utilise the analytic properties of oneloop amplitudes to generate the coefficients of one-loop integral functions. By combining these carefully, we have generated explicit expressions for the coefficients of six and seven-point three-mass triangle functions in $\mathcal{N}=1$. These expressions provide an excellent springboard for generating all $n$-expressions. 


\section{Acknowledgments}

We wish to thank Zvi Bern, Lance Dixon and especially Harald Ita for many helpful conversations. This research was supported in part by the Science and Technology Facilities Council of the UK and in part by grant DE-FG02-90ER40542 of the US Department of Energy. NEJBB would like to thank the Niels Bohr Institute and Swansea University for hospitality during the completion of this work.

\section{References}

[1] R. P. Feynman, Phys. Rev. 76 (1949) 749, Phys. Rev. 76 (1949) 769;

G. 't Hooft and M. J. G. Veltman, NATO Adv. Study Inst. Ser. B Phys. 4 (1974) 177.

[2] G. Passarino and M. J. G. Veltman, Nucl. Phys. B 160 (1979) 151;

R. G. Stuart, Comput. Phys. Commun. 48 (1988) 367;

G. J. van Oldenborgh and J. A. M. Vermaseren, Z. Phys. C 46 (1990) 425.

[3] R. K. Ellis, W. T. Giele and G. Zanderighi, JHEP 0605 (2006) 027 [hep-ph/0602185].

[4] A. Denner, S. Dittmaier, M. Roth and L. H. Wieders, Phys. Lett. B 612 (2005) 223 [hep-ph/0502063];

T. Binoth, J. P. Guillet, G. Heinrich, E. Pilon and C. Schubert, JHEP 0510 (2005) 015 [hep-ph/0504267];

G. Belanger, F. Boudjema, J. Fujimoto, T. Ishikawa, T. Kaneko, K. Kato and Y. Shimizu, Phys. Rept. 430 (2006) 117 [hep-ph/0308080].

[5] E. Witten, Commun. Math. Phys. 252, 189 (2004) [hep-th/0312171].

[6] Z. Bern, L.J. Dixon, D.C. Dunbar and D.A. Kosower, Nucl. Phys. B 425, 217 (1994), [hep-ph/9403226].

[7] Z. Bern, L.J. Dixon, D.C. Dunbar and D.A. Kosower, Nucl. Phys. B 435, 59 (1995), [hep-ph/9409265].

[8] Z. Bern, L. J. Dixon and D. A. Kosower, Nucl. Phys. B 513 (1998) 3 [hep-ph/9708239].

[9] R. Britto, E. Buchbinder, F. Cachazo and B. Feng, Phys. Rev. D 72 (2005) 065012 [hep-ph/0503132].

[10] R. Britto, B. Feng and P. Mastrolia, Phys. Rev. D 73 (2006) 105004 [hep-ph/0602178].

[11] R. K. Ellis, W. T. Giele and Z. Kunszt, [hep-ph/0708.2398];

S. D. Badger, E. W. N. Glover and K. Risager, JHEP 0707, 066 (2007) [hep-ph/0704.3914]. 
[12] W. L. van Neerven, Nucl. Phys. B 268 (1986) 453;

Z. Bern and A. G. Morgan, Nucl. Phys. B 467 (1996) 479 [hep-ph/9511336];

Z. Bern, L. J. Dixon, D. C. Dunbar and D. A. Kosower, Phys. Lett. B 394 (1997) 105 [hep-th/9611127];

A. Brandhuber, S. McNamara, B. J. Spence and G. Travaglini, JHEP 0510, 011 (2005) [hep-th/0506068];

C. Anastasiou, R. Britto, B. Feng, Z. Kunszt and P. Mastrolia, Phys. Lett. B 645 (2007) 213 [hep-ph/0609191];

C. Anastasiou, R. Britto, B. Feng, Z. Kunszt and P. Mastrolia, JHEP 0703 (2007) 111 [hep-ph/0612277].

[13] R. Britto, F. Cachazo and B. Feng, super-Yang-Mills," Nucl. Phys. B 725 (2005) 275 [hep-th/0412103].

[14] S. J. Bidder, N. E. J. Bjerrum-Bohr, D. C. Dunbar and W. B. Perkins, Phys. Lett. B 612 (2005) 75 [hep-th/0502028].

[15] F. Cachazo, hep-th/0410077.

[16] R. Britto, F. Cachazo and B. Feng, Phys. Rev. D 71 (2005) 025012 [hep-th/0410179].

[17] S. J. Bidder, N. E. J. Bjerrum-Bohr, L. J. Dixon and D. C. Dunbar, Phys. Lett. B 606, 189 (2005) [hep-th/0410296].

[18] G. Ossola, C. G. Papadopoulos and R. Pittau, Nucl. Phys. B 763, 147 (2007) [hep-ph/0609007];

G. Ossola, C. G. Papadopoulos and R. Pittau, JHEP 0707 (2007) 085 [hep-ph/0704.1271].

[19] D. Forde, Phys. Rev. D 75 (2007) 125019 [hep-ph/0704.1835].

[20] Z. Bern, N. E. J. Bjerrum-Bohr, D. C. Dunbar and H. Ita, JHEP 0511 (2005) 027 [hep-ph/0507019].

[21] Z. Bern and D. A. Kosower, Nucl. Phys. B 362 (1991) 389.

[22] H. J. Lu and C. A. Perez, SLAC-PUB-5809, May 1992.

[23] Z. Bern, L. J. Dixon and D. A. Kosower, Nucl. Phys. B 412 (1994) 751 [hep-ph/9306240].

[24] Z. Xu, D. H. Zhang and L. Chang, Nucl. Phys. B 291, 392 (1987).

[25] S. J. Bidder, N. E. J. Bjerrum-Bohr, D. C. Dunbar and W. B. Perkins, Phys. Lett. B 608 (2005) 151 [hep-th/0412023].

[26] P. Mastrolia, Phys. Lett. B 644 (2007) 272 [hep-th/0611091]. 
[27] N. E. J. Bjerrum-Bohr, D. C. Dunbar, H. Ita, W. B. Perkins and K. Risager, JHEP 0612 (2006) 072 [hep-th/0610043];

Z. Bern, N. E. J. Bjerrum-Bohr and D. C. Dunbar, JHEP 0505 (2005) 056 [hep-th/0501137];

Z. Bern, J. J. Carrasco, D. Forde, H. Ita and H. Johansson, [hep-th/0707.1035].

[28] R. Britto, F. Cachazo and B. Feng, Nucl. Phys. B 715 (2005) 499 [hep-th/0412308];

R. Britto, F. Cachazo, B. Feng and E. Witten, Phys. Rev. Lett. 94 (2005) 181602 [hep-th/0501052];

C. F. Berger, Z. Bern, L. J. Dixon, D. Forde and D. A. Kosower, Phys. Rev. D 74 (2006) 036009 [hep-ph/0604195];

Z. Bern, L. J. Dixon and D. A. Kosower, Phys. Rev. D 73 (2006) 065013 [hep-ph/0507005]. 